\title{
Wideband Code Division Multiple Access
}

\author{
Laurence B. Milstein, Fellow, IEEE
}

Invited Paper

\begin{abstract}
To satisfy ever-increasing demands for higher data rates, as well as to allow more users to simultaneously access the network, interest has peaked in what has come to be known as wideband code division multiple access (WCDMA). In this paper, we discuss those basic characteristics of WCDMA waveforms that make them attractive for high data rate transmission over wireless and mobile channels. We emphasize how the choice of spread bandwidth affects the bit error rate of the system, as well as how it affects the reliability of various subsystems, such as those that perform coarse acquisition and adaptive power control. We then discuss how some of the waveforms being considered for cellular WCDMA systems differ from those in use in the narrowband CDMA cellular system, and emphasize, as an example, multicarrier CDMA. Finally, we discuss other potential enhancements to WCDMA systems, such as the use of interference suppression at the receiver, or multiple antennas at the transmitter.
\end{abstract}

Index Terms-Code division multiple access (CDMA), spread spectrum communicating.

\section{INTRODUCTION}

$\mathbf{I}$ N THIS partially tutorial paper, the key attributes and waveform design considerations of a direct sequence (DS), wideband, code division multiple access (CDMA) system, when used over a wireless channel, are discussed. As is well known, the use of direct sequence spread spectrum to combat potential sources of degradation, such as multipath fading, is a technique that has been known for decades (see [7], [16], [24], [28], [47], [57], [58], [78] for representative references). Similarly, the use of DS for CDMA is decades old [4], [5], [8], [9], [39], [59], [74], [79]. However, this paper is primarily concerned with developments that occurred over the last decade or so, because it was over that time frame that debates over the relative characteristics of wideband and narrowband CDMA became focused. Indeed, prior to that time frame, adjectives such as "narrowband" and "wideband" were rarely, if ever, used in the designation of a specific CDMA system.

Note that there is no single, universally accepted, definition of wideband CDMA, and, in fact, definitions based upon system parameters such as chip rate, or bandwidth as a fraction of center

\footnotetext{
Manuscript received August 1999; revised February 28, 2000. This work was supported in part by the Army Research Office under Grant DAAG55-98-10473, and the Office of Naval Research under Grant N00014-98-1-0875.

The author is with the Department of Electrical and Computer Engineering, University of California, San Diego, La Jolla, CA 92093-0407 USA (e-mail: milstein@ece.ucsd.edu).

Publisher Item Identifier S 0733-8716(00)06104-7.
}

frequency, are both intuitive and useful in various scenarios. However, for the purposes of this paper, we will use a definition more intimately tied to the distinction be wideband and narrowband CDMA when used over a wireless channel. Specifically, a wideband CDMA (WCDMA) system is defined as one wherein the spread bandwidth of the underlying waveforms in the system typically exceed the coherence bandwidth of the channel over which the waveforms are transmitted (meaning irrespective of whether the channel is indoors, outdoor urban, outdoor suburban, etc.). This results in the channel appearing frequency-selective to those waveforms, and correspondingly results in enhanced system performance relative to that of a narrowband CDMA system, wherein the latter is defined as one which (too often) experiences flat fading. The justification, for the previous statement, is that a direct sequence system which experiences frequency-selective fading can, via the use of a rake receiver, resolve individual multipath components and then coherently combine them (i.e., it can make constructive use of the multipath). Finally, there is, of course, no distinct bandwidth threshold that separates narrowband CDMA from WCDMA. However, based upon measured data in references such as [32] and [50], it appears that a spread bandwidth of about $10 \mathrm{MHz}$ satisfies the intuitive requirement that the channel appear frequency selective to the transmitted waveform for a broad crosssection of scenarios.

In what follows, emphasis is placed on the underlying concepts, not on specific implementations being suggested for various proposed WCDMA systems. Also, the term WCDMA is used generically, and is not tied to the name of any particular system design. For those readers who are interested in the specifics of actual WCDMA designs, [76] and [77] present multiple papers on the role of WCDMA in the standards process referred to as "IMT 2000." Among the topics to be discussed below are the use of both multicarrier CDMA and multicode CDMA, CDMA system design for connectionless packet transmission, and the use of multiple access interference suppression techniques. However, before delving into such specific topics, an overview of the evolution of WCDMA is presented, along with a more in-depth look at its basic characteristics.

\section{THE ORIGINS OF WCDMA}

Spread spectrum communications systems, both direct sequence and frequency hopped, have been in existence for decades, although up until the last decade or so, most of those systems have been military systems, where the need for signals 
displaying anti-jam and low probability-of-intercept characteristics was paramount. Thus, they were typically designed to be wideband, and those that employed DS to achieve multiple access capabililty were the original forerunners of what is now called wideband CDMA. In the late 1980s, the use of DS CDMA started to become increasingly of interest to the commercial sector for use in cellular-type communications, and both narrowband CDMA and wideband CDMA systems were designed. In this latter time frame, what appear to be the first narrowband and the first wideband CDMA systems to be proposed were those described in [73] and [69], respectively, where the wideband system of [69] was originally envisioned as an overlay of conventional microwave signals in the 1850-1990 $\mathrm{MHz}$ region.

That overlay system employed a spread bandwidth larger than even the largest of the currently projected WCDMA bandwidths. The initial WCDMA cellular systems are envisioned to spread over $5 \mathrm{MHz}$, and future WCDMA systems are projected to spread over either 10 or $20 \mathrm{MHz}$. In contrast, what was then referred to as a "broadband" CDMA system used a 24 Mchip/s waveform occupying about $48 \mathrm{MHz}$ of bandwidth. The fundamental reason for such a large spread bandwidth was the desire to overlay fixed-service microwave systems (i.e., to allow the spread spectrum network to share common bandwidth with the narrowband microwave signals). This resulted in the spread bandwidth being much larger than the coherence bandwidth of the channel, and thus the DS signals experienced relatively little fading [49], [50], [70].

The overlay proposal was introduced in 1989 and, since that time, both field tests and analyses have provided a perspective as to what the capabilities of such a system are. Indeed, the overlay was demonstrated in the PCS band and also in the cellular band (whereby, in the latter case, the overlayed waveforms were AMPS signals). In both cases, it was found that, in order to successfully deploy the overlay, i.e., to deploy it in a manner such that neither set of users caused excessive interference to the other set, it was desirable to use tunable notch filters at both the CDMA transmitters and the CDMA receivers. By placing narrow notches at the CDMA transmitters corresponding to those locations where the narrowband waveforms are situated, interference to those narrowband signals is reduced. Similarly, implementing notches at the CDMA receivers at the locations of the narrowband waveforms decreases interference to the CDMA network.

As a consequence, there have been many studies on the role of notch filters in an overlay situation; the results of some of those studies can be found in references such as [15], [26], [31], [66], [72], [83], and [84]. Recently, a new approach has been suggested [42]; this latter technique makes use of a multicarrier (MC) CDMA waveform. In such a design, multiple narrowband DS CDMA waveforms, each at a distinct carrier frequency, are combined to yield a composite wideband CDMA signal. Among the advantages of such an approach is the ability to achieve the same type of system performance that a conventional, single carrier, wideband CDMA signal would provide, such as diversity enhancement over a multipath channel; however, this is achieved without the need for a contiguous spectral band over which to spread. Thus, regarding the overlay, MC transmission can be especially attractive, since frequency slots occupied by narrowband waveforms can be avoided altogether by simply not transmitting at the corresponding carrier frequencies. This is discussed more fully in Section IV-B.

From the results of the performance of a CDMA system employing such a broadband waveform when used on multipath fading channels, interest evolved in deploying a very wideband CDMA network, although without the overlay. Rather, the motivation for what came to be known as WCDMA was both increased information rate, so as to ensure the capability of transmitting multimedia traffic, and enhanced robustness to multipath fading, an attribute which ultimately results in higher capacity. In particular, researchers such as those at NTT DoCoMo [17], [18] demonstrated the desirable properties of a WCDMA signal when used over a multipath fading channel, and their results reinforced those of [50], as well as reinforcing those of numerous predecessors (e.g., [16], [24], and [28]).

\section{CHARACTERISTICS OF WCDMA WAVEFORMS}

The key attribute of a very wideband direct sequence signal is its ability to resolve individual multipath components and, through the mechanism of a rake receiver, coherently combine them. While much literature exists on the performance of CDMA systems over frequency-selective channels (see, e.g., [9], [53], [54], [82]), in what follows, we concentrate primarily on the effect of spread bandwidth as it relates to comparative performance of wideband and narrowband systems, as can be found in references such as [17], [20]-[22], [30], and [52].

\section{A. Bit-Error Rate Considerations}

As is well known, if one wants a DS system to operate effectively over a multipath channel, the channel should appear frequency-selective to the waveform; stated differently, the spread bandwidth should exceed the coherence bandwidth of the channel. This allows a rake receiver to be employed so that multiple reflections of the transmitted waveform can be resolved and then coherently combined. However, there is a tradeoff in this type of receiver design, in that as the spread bandwidth increases, thus allowing more paths to be resolved, the energy-per-resolved path decreases, thus making it more difficult for the receiver to estimate the amplitudes and phases of the paths as required by an optimal rake receiver.

To be specific, in [20], the tradeoff discussed above is described, and, among other things, it is shown that when coherent combining is employed, along with perfect channel estimates, the wideband system always outperforms the narrowband system. On the other hand, this conclusion does not necessarily follow if noncoherent combining is used. These results can be explained as follows. Considering first a coherent receiver, for a wideband system whereby each user spreads its signal over the entire bandwidth, the number of resolvable paths available for combining is maximized, and the coherent combining of those paths results in the most efficient use of the desired signal's energy. If, instead of using the above approach, one uses a hybrid scheme, whereby the total bandwidth is first divided into smaller segments and those segments individually support narrowband CDMA networks (i.e., if one uses an 


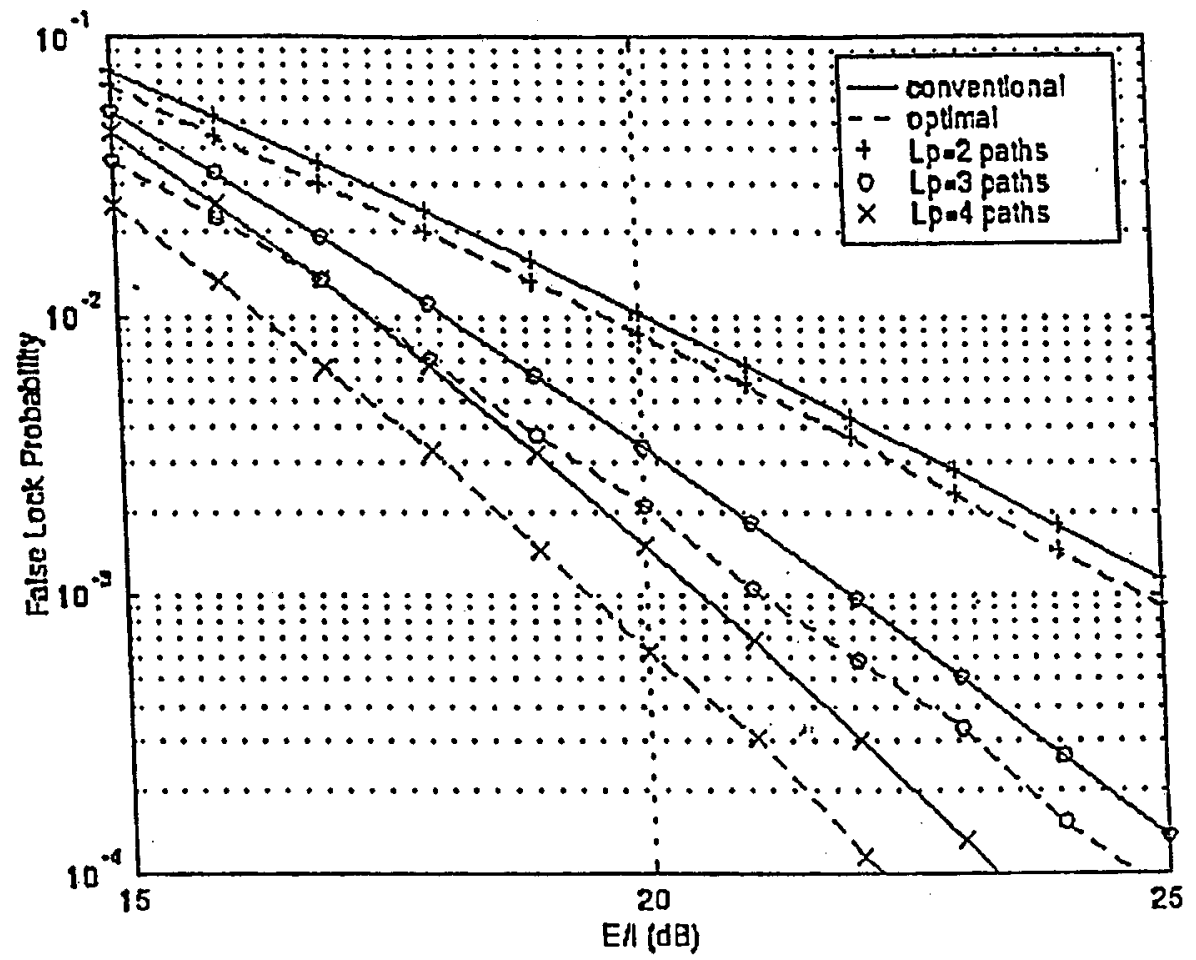

Fig. 1. Comparison of optimal and conventional parallel acquisition schemes.

FDMA/CDMA architecture), the ultimate benefits of the rake combining are not achieved.

Alternatively, if noncoherent reception is employed with WCDMA, as more and more paths are combined, the system experiences a noncoherent combining loss. Thus, depending upon the signal-to-noise ratio and the amount of interference seen by the receiver, there are scenarios for which a hybrid system yields better performance than one which spreads across the entire available bandwidth, and specific examples are presented in [20]. Additional results that illustrate the tradeoff in performance with spread bandwidth when a noncoherent modulation format is employed can be found in [30].

Returning to a coherent system, while results such as those presented in [20] are useful, they do not provide a complete picture, because they are based upon perfect estimation of the channel parameters. To remove that idealization, the analyses of [21] and [22] show what happens when imperfect phase estimates are used. These latter results, obtained analytically by approximate bounding techniques in [21], and by simulations in [22], illustrate that, indeed, as the signal-to-noise ratio (SNR) becomes lower, the ability to accurately estimate the phase decreases, and thus a tradeoff in overall system performance exists between WCDMA and hybrid FDMA/CDMA, analogous to the tradeoff if noncoherent reception is employed. However, as can be seen in ([22], Table III), one needs a reasonably low SNR in the tracking loop before a hybrid system outperforms a wideband system.

Finally, for a different perspective on the advantage of a wider spread bandwidth, consider the experimental curves presented in the tutorial paper by Adachi, Sawahashi, and Suda [3] (see, specifically, Figs. 14 and 15 of [3]). Results are presented for the chip rates ranging from $0.96 \mathrm{Mchips} / \mathrm{s}$ to $7.68 \mathrm{Mchips} / \mathrm{s}$.
As discussed in [3], the required transmit power to achieve a given level of performance decreases as the chip rate (and hence the spread bandwidth) increases. As an example, for the system tested in [3], increasing the chip rate from 0.96 Mchips/s to 3.04 Mchips/s results in a $1.2 \mathrm{~dB}$ reduction in the median transmit power, and increasing it further to $7.68 \mathrm{Mchips} / \mathrm{s}$ results in an additional $4 \mathrm{~dB}$ reduction. Other experimental results presented in [3] show the reduction in probability of error that is achievable when increasing the chip rate. For example, at a ratio of energy-per-bit-to-interference spectral density of $10 \mathrm{~dB}$, going from $0.96 \mathrm{Mchips} / \mathrm{s}$ to $7.68 \mathrm{Mchips} / \mathrm{s}$ reduced the probability of error by about an order of magnitude for the system under test.

\section{B. Acquisition}

Note that the results referred to in Subsection A are based upon bit-error rate considerations, and most of them correspond to system performance under the assumption of perfect acquisition and tracking of the spreading sequences. In [61] and [62], the coarse acquisition problem is addressed, and it is shown that, as is the case if BER is the criterion, wideband spreading provides better performance than does narrowband spreading. Since a wideband DS waveform has multiple correct paths to which one can lock (i.e., if there are, say, $L$ resolvable multipath components, then there are that many correct phase positions), the likelihood of observing a correct path during the search process has been increased from what it would be for narrowband CDMA, which corresponds to $L=1$. To be specific, consider results from [62], in which an optimal strategy is derived for a parallel coarse acquisition receiver. Shown in Fig. 1 are several sets of curves, where, in each set, there is one curve showing the performance of a conventional receiver (see 


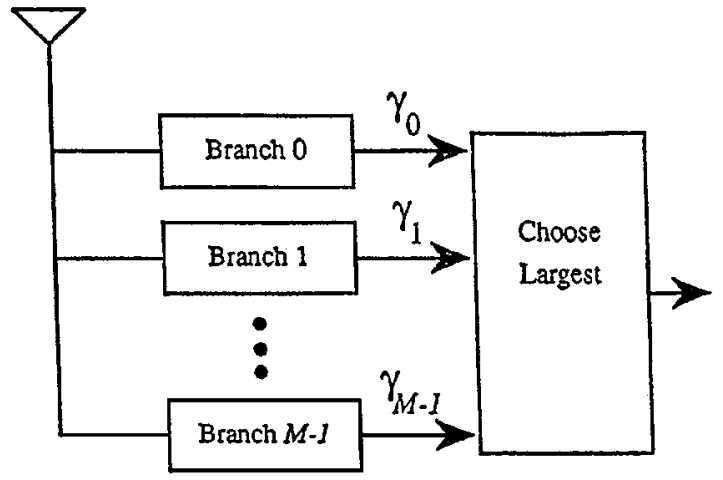

(a)

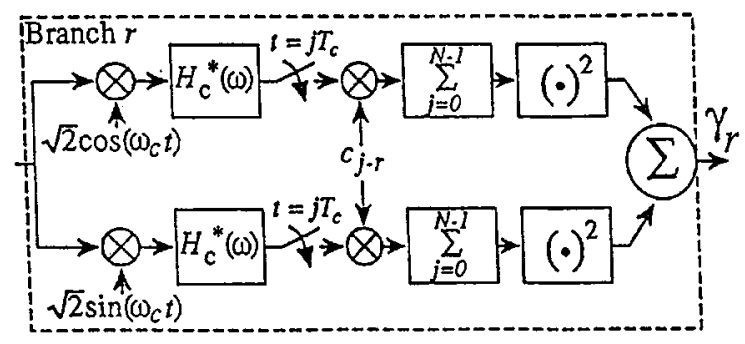

(b)

Fig. 2. (a) Conventional parallel acquisition receiver. (b) In-phase/quadrature noncoherent detector.

Fig. 2) and one showing performance of the optimal receiver, corresponding to a test statistic more complex than the one used in Fig. 2 (the interested reader can find it in [62]). Note that the sets of curves are parameterized by the number of resolvable paths. Interestingly, it is seen that, depending on the number of resolvable paths, the conventional receiver performs fairly well relative to the optimal receiver. However, if one is interested in enhancing acquisition performance, the more advantageous way to achieve it is to increase the spread bandwidth in the CDMA system, as opposed to implementing a more complex receiver structure at a given spread bandwidth. For example, consider the curves of Fig. 1 corresponding to two resolvable paths. If the target false lock probability is, say, $10^{-3}$, the performance of the optimal receiver is several tenths of a decibel better than that of the conventional receiver. However, if the spread bandwidth is doubled, so that now four paths are resolvable, the performance gain is somewhere between 4 and $5 \mathrm{~dB}$. One could also gain an extra decibel or so of improvement if simultaneously the spread bandwidth is doubled and the optimal receiver is used, but most of the total improvement comes from the use of the wider spread bandwidth.

\section{Power Control}

The same general conclusions as discussed above also apply to power control, as illustrated in [11], [12], and [30]. For this latter consideration, there are two key effects taking place, and each is favorable to the wideband system. First, the accuracy with which a power measurement can be made increase with increasing spread bandwidth, since to amount of fading the signal experience decreased (see [11]). Second, the effect of any residual measurement inaccuracy is less in a wideband system than it is in a narrowband system; that is, for a given power control error, the decrease in capacity experienced by a narrowband CDMA system is greater than that seen by a WCDMA system. Note that in a multicell system, even with perfect power control, one would expect WCDMA to be superior. This follows because narrowband CDMA experiences more attenuation due to multipath fading than does WCDMA. Hence, even if the power control is perfect, the additional power that must be transmitted by a narrowband CDMA system (to overcome the increased loss due to multipath fading) spills over into surrounding cells, and thus causes increased intercell interference.

\section{Fading Statistics}

Finally, there is one other key characteristic that should be pointed out. As observed, for example, in [36], [40], [41], [51], when a DS signal is received over a multipath fading channel, the amplitude distribution of the despread signal becomes more and more specular as the spread bandwidth increases. This is irrespective of the fading statistics of the received waveform. For example, assume the received signal on any given resolvable path is experiencing Rayleigh fading at the input to a despreader that is synchronized to that path. That particular resolvable path is, in turn, the vector sum of many physical paths, and it is the constructive/destructive interference patterns resulting from that vector summation that results in a Rayleigh distribution for the amplitude of the received signal. Note that we make a distinction between physical paths, which is a property of the channel, and resolvable paths, which is a property of the channel, the waveform design, and the receiver. When the signal is now despread, only a fraction of those multiple paths offer any significant contribution to the output (because the remaining paths experience a large attenuation due to the sharply localized autocorrelation function of a typical spreading sequence). Indeed, as the spread bandwidth increases, the autocorrelation function becomes more localized, and a larger number of paths are attenuated to the extent that they become insignificant. Thus, the despreader output becomes dominated by a decreasing number of paths (as spread bandwidth increases), and no longer is characterized by a Rayleigh probability distribution; rather, its statistics have been found to be more closely described by a Rician density. This, of course, suggests, that the effect of the estimation errors discussed in Subsection A is overly pessimistic, since the analyses that produced those results are based upon Rayleigh statistics, independent of the spread bandwidth.

\section{WCDMA WAVEFORM DESIGN ALTERNATIVES FOR Cellular Systems}

This section describes some of the signal designs and receiver structures that are being considered for WCDMA systems.

\section{A. Coherent Reverse Link}

One design change relates to the signal structure on the mobile-to-base link, commonly referred to as the reverse link. Whereas virtually all of the initial implementations of the NCDMA system of [73] use noncoherent detection on the reverse link (specifically, they use 64-ary orthogonal signaling with noncoherent detection), the current WCDMA waveform designs for the reverse link employ either BPSK or QPSK with 


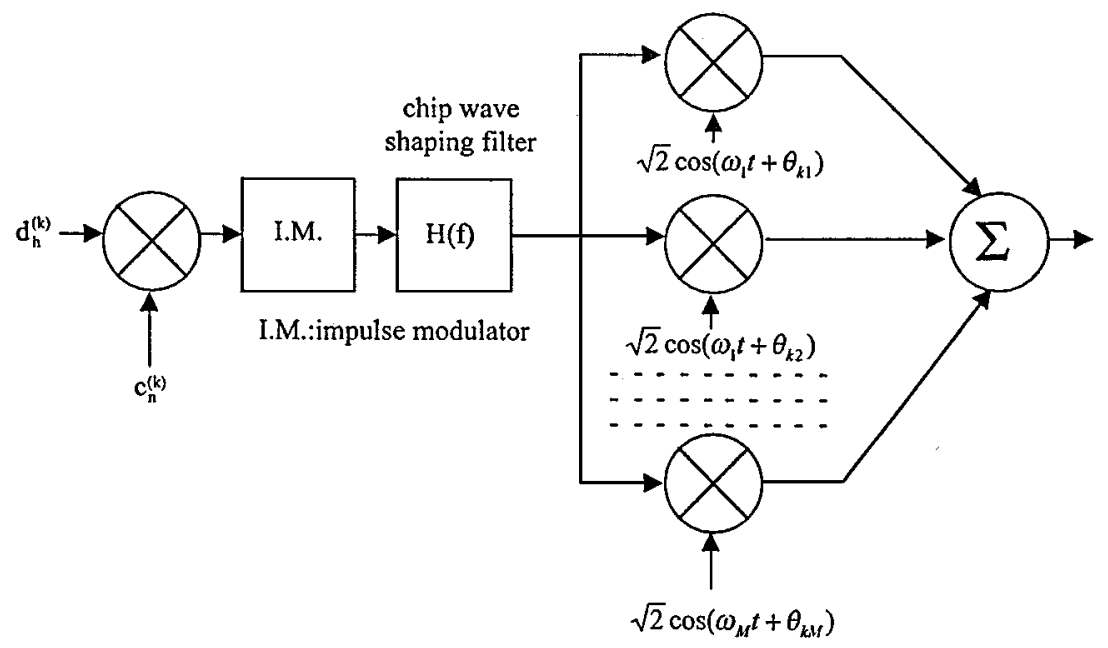

Fig. 3. The $k$ th transmitter.

coherent detection. This type of reverse link design is what was proposed for the CDMA system of [50], and is consistent with earlier designs for DS spread spectrum (e.g., [24], [28], and [39]).

\section{B. Multicarrier CDMA}

Another proposed change in the waveform design is the consideration of a multicarrier (MC) signal for the forward link (i.e., the link from the base to the mobile). The driving force for such a waveform design is the ease with which one can convert from a narrowband CDMA signal to a wideband CDMA signal. That is, by taking, say, $M$ narrowband CDMA waveforms, each on a different carrier frequency, and assigning them all to one user, one can now increase the spread bandwidth by a factor of $M$. There are various ways in which one can create an MC signal, and, indeed, there is no uniformly accepted definition of $\mathrm{MC}$ CDMA. For example, some define MC CDMA as a combination of orthogonal frequency division multiplexing (OFDM) and CDMA, where the number of carriers typically equals the processing gain ([10], [13], [25], [88]). However, we will use the $\mathrm{MC}$ architecture of [42], corresponding to the parallel transmission of multiple narrowband DS waveforms, where the number of such waveforms is typically much less than the processing gain. The reason for this is the likelihood that this latter model or, more precisely, a coded version of it, as in [63], will be the first to be deployed in a WCDMA design.

To be specific, we consider a direct sequence spread spectrum system in which a data sequence multiplied by a spreading sequence modulates $M$ carriers (see, e.g., [14], [42]-[46], [60], [63]-[65], [75], [85], [86], [89]). Further, we consider the reverse link as well as the forward link. The receiver provides a correlator for each carrier, and the outputs of the correlators are combined to yield a processing gain comparable to that of a single carrier DS system. This type of system has the following characteristics. Similar to a conventional single carrier DS system, a multicarrier DS spread spectrum system is robust to multipath fading, as long as the total spread bandwidth exceeds the channel coherence bandwidth. The reason for this will be seen below. Also, as mentioned in Section II, a multicarrier system has a narrowband interference mitigation effect, and this will be more explicitly illustrated below. Further, in a multicarrier DS system with $M$ carriers, the entire bandwidth of the system is divided into $M$ equiwidth frequency bands, and thus each carrier frequency is modulated by a spreading sequence with a chip rate which is $1 / M$ times that of a single carrier system. Thus, a multicarrier system requires a lower speed, parallel-type of signal processing, in contrast to a fast, serial-type of signal processing in a single carrier rake receiver.

In essence, the use of this type of multicarrier design results in a tradeoff of the explicit path diversity one would achieve by using a single carrier DS waveform (with the same total spread bandwidth) for the explicit frequency diversity achievable with the multicarrier waveform. Further, if one views the transmitter shown in Fig. 3, in which $\omega_{m}$ is the $m$ th carrier frequency, and $\theta_{k, m}$ is a random phase associated with the $m$ th subcarrier of the $k$ th user, as the equivalent of a rate $1 / M$ repetition code, then one can easily envision using a more sophisticated code, such as a convolutional code [63]-[65], rather than simply repeating the same data symbol on each of the $M$ subcarriers.

Note that there are many considerations in the choice of the parameter $M$. If we start out with a baseline system consisting of an MC system wherein the bandwidth of each of the carriers equals the coherence bandwidth of the channel, then increasing $M$ to some value, say, $M 1>M$, results in correlated fading among the carriers, the consequence of which is an effective diversity order less than $M 1$. Alternately, decreasing $M$ to some other value, say, $M 2<M$, results in frequency-selective fading on each carrier, and thus a rake receiver on each subcarrier is required in order to achieve optimal performance.

Similarly, increasing $M$ results in increased coding gain, but decreased processing gain per subcarrier. Also, increasing $M$ results in greater complexity at the transmitter, and, to the extent that the transmitter has a saturating power amplifier, increasing $M$ results in more intermodulation products among the carriers. These and other considerations are discussed in references such as [85] and [86].

1) Performance of $M C$ CDMA: Consider now a more quantitative comparison of $\mathrm{MC}$ and single carrier CDMA, taken from [43], and corresponding to the reverse link. Fig. 4(a) shows a bandlimited single carrier wideband DS waveform in the fre- 


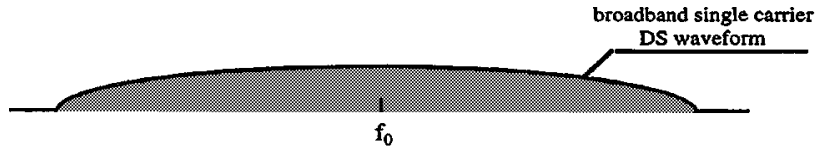

(a) spectrum single carrier DS waveform

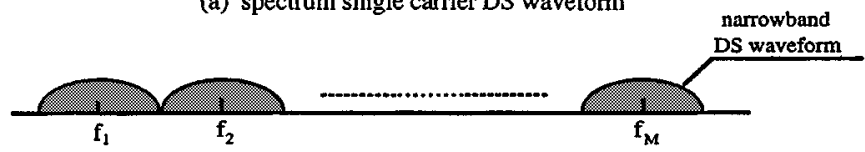

(b) spectrum of multicarrier DS waveform

Fig. 4. Power spectral densities of single carrier and multicarrier waveforms.

quency domain. The bandwidth, denoted $\mathrm{BW}_{1}$, is given by $(1+$ $\alpha) / T_{c}$, where $\alpha$ is the excess bandwidth of the system and $1 / T_{c}$ is the chip rate of the single carrier system. In a multicarrier system, we divide $\mathrm{BW}_{1}$ into $M$ equiwidth frequency bands, as shown in Fig. 4(b), where all bands are disjoint. Then the bandwidth of each frequency band, $\mathrm{BW}_{M}$, is given by $(1+\alpha) / M T_{c}$. Note that $1 / M T_{c}$ is the chip rate of the multicarrier system.

For the $k$ th transmitter shown in Fig. $3, d_{h}^{(k)}$ is a random binary sequence representing the data, and $c_{n}^{(k)}$ is a signature sequence. Each of the $M$ spread spectrum signals has $N$ chips per symbol, and each user has a different signature sequence. Conceptually, the sequence $d_{h}^{(k)} c_{n}^{(k)}$ modulates an impulse train, where the energy-per-chip is $E_{c}$. After passing through a chip wave-shaping filter, the signal out of the filter modulates the multiple carriers and is transmitted.

The channel is assumed to be a slowly varying, frequency-selective, Rayleigh channel with delay spread of $T_{m}$, where we define the delay spread to be the range of delays over which the multipath intensity profile is essentially nonzero. In order to facilitate a performance comparison between a single carrier rake system and the multicarrier system, we define two channel models; one is a time domain model for a rake system, and the other is a frequency domain model for a multicarrier system. The former model is used to emphasize the path diversity achievable with a single carrier waveform, and the latter model emphasizes the frequency diversity achievable with multicarrier waveforms.

For a single carrier system, the key parameters are the processing gain, $N_{1}$, which equals $M N$, and the number of resolvable paths of the channel, which we denote as $L_{1}$, and which is given by $L_{1}=\left\lfloor T_{m} / T_{c}\right\rfloor+1$, where $\lfloor x\rfloor$ denotes the integer part of $x$. Then the complex, lowpass, equivalent impulse response of the channel can be modeled as

$$
c(t)=\sum_{\ell=0}^{L_{1}-1} \Psi_{\ell} \delta\left(t-\ell T_{c}\right)
$$

where $\Psi_{\ell}$ is a zero-mean, complex Gaussian random variable, and $\delta(t)$ is a Dirac delta function. Note that we are assuming that the channel contains as many resolvable paths as the waveform is capable of resolving.

On the other hand, a frequency domain channel model can be characterized by the coherence bandwidth, $(\Delta f)_{c}$, which is given by $(\Delta f)_{c} \approx 1 / T_{m}$. For the analysis below, we choose the number of carriers in the MC system to equal the number of resolvable paths in the single carrier system, so that $M=L_{1}$.

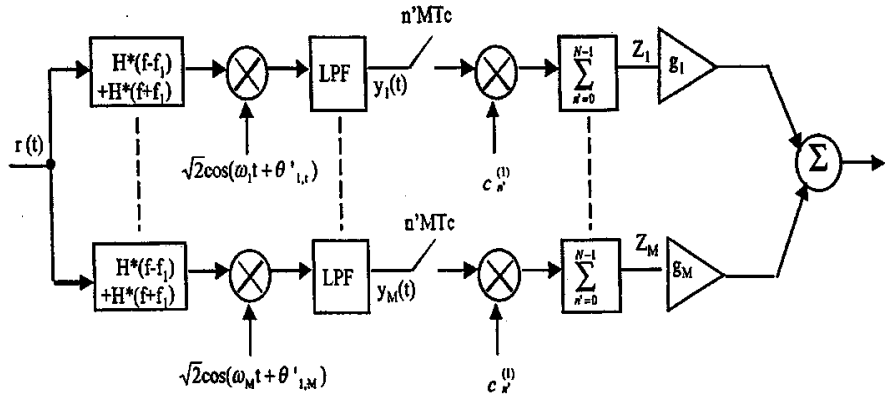

Fig. 5. Receiver for the first signal.

This makes precise the statement made above that the use of MC rather than single carrier results in a tradeoff of path diversity for frequency diversity.

The complex lowpass equivalent impulse response of the $i$ th channel can be written as $c_{i}=\varsigma_{i} \delta(t), i=1 \ldots M$, where the $\left\{\varsigma_{i}, i=1 \ldots M\right\}$ are i.i.d. zero-mean, complex Gaussian random variables. Then the transfer function of the channel spanned by the $i$ th frequency band for the $k$ th user is given by $\varsigma_{k, i} \equiv \alpha_{k, i} \exp \left(j \beta_{k, i}\right)$, where the $\left\{\alpha_{k, i}\right\}$ and $\left\{\beta_{k, i}\right\}$ are, respectively, i.i.d. Rayleigh random variables, each with a unit second moment, and i.i.d. uniform random variables over $(0,2 \pi)$.

The received signal is given by

$$
\begin{aligned}
r(t)= & \sum_{k=1}^{K}\left\{\sqrt{2 E_{c}} \sum_{n=-\infty}^{\infty} d_{n}^{(k)} c_{n}^{(k)} h\left(t-n M T_{c}-\tau_{k}\right)\right. \\
& \left.\times \sum_{m=1}^{M} \alpha_{k, m} \cos \left(\omega_{m} t+\theta_{k, m}^{\prime}\right)\right\}+n_{w}(t)+n_{J}(t)
\end{aligned}
$$

where $\hat{n}=\lfloor n / N\rfloor, h(t)$ is the impulse response of the chip wave-shaping filter, $\theta_{k, m}^{\prime}=\theta_{k, m}+\beta_{k, m}, n_{w}(t)$ is white Gaussian noise with a double-sided psd of $\eta_{0} / 2$, and $n_{J}(t)$ represents narrowband, bandpass interference that is assumed to be Gaussian with a bandlimited double-sided power spectral density of $n_{J} / 2$, center frequency of $f_{J}$, and a bandwidth of $W_{J} \mathrm{~Hz}$. Such an interfering signal might, for example, represent a narrowband waveform to be overlaid by the wideband MC CDMA network.

The receiver of the first user $(k=1)$ is shown in Fig. 5, where we assume the chip wave-shaping filter is a square-root raised-cosine Nyquist filter with an excess bandwidth of $\alpha$. Note that none of the multiple waveforms that make up the MC signal overlaps with one another. Also, the gains $\left\{g_{i}\right\}$ in Fig. 5 are the coefficients needed for maximal-ratio combining. For the results presented below, they are assumed to be perfectly known.

Fig. 6 shows the probability of error versus $E_{b} / \eta_{0}$ for $K=$ 50 , where $K$ is the number of signals and $E_{b}$ is the energy per bit. The curves are parameterized by $J S R$, where $J S R$ is given by

$$
J S R=\frac{\text { interference power }}{\text { signal power }}=\frac{\eta_{J} W_{J}}{E_{b} / T} .
$$

The results correspond to the interfering waveform overlapping the second DS signal of the multicarrier system, i.e., $f_{J}=f_{2}$ 


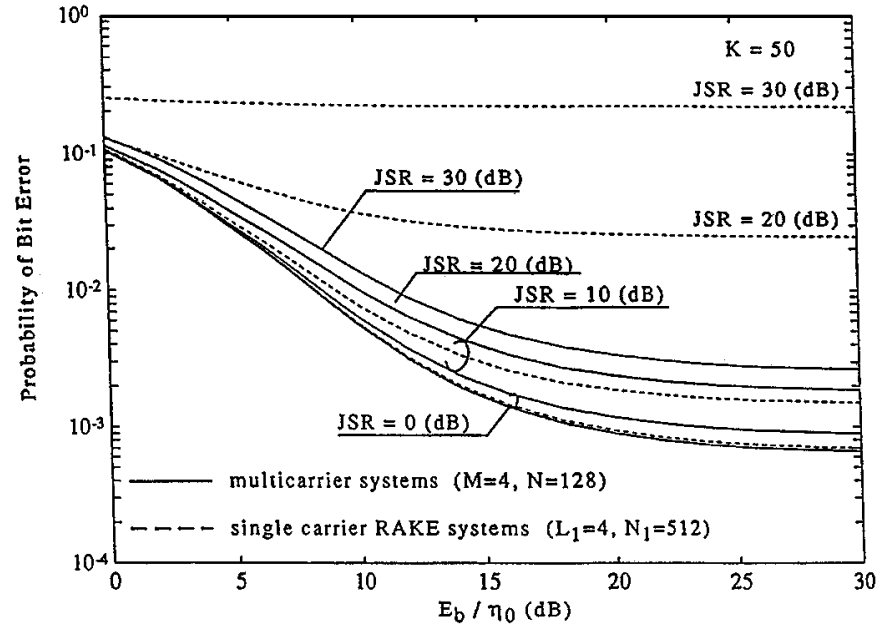

Fig. 6. BER versus $E_{b} / \eta_{0}$ for $K=50, f_{J}=f_{2}, W_{J}=\mathrm{BW}_{M}$, and $\alpha=0.5$.

and $W_{J}=\mathrm{BW}_{M}$. If $J S R$ is small, the performance of a single carrier rake system and that of a multicarrier system are almost the same. However, we see that the multicarrier system outperforms the single carrier system if $J S R$ is large. This is because the multicarrier system has, for this example, interference only in the second frequency band and, as a result, $g_{2}$ in Fig. 5 becomes very small for large interference power. In this manner, the use of a MC signal design can accomplish an efficient CDMA overlay, as discussed in references such as [44] and [60].

2) Forward Link Considerations: In the above discussion on multicarrier CDMA, all the results correspond to the reverse link of a cellular system (i.e., they correspond to an asynchronous CDMA system). To end this subsection, consider the use of a multicarrier waveform on the forward link, which is actually a multiplexed link, rather than a multiple access link. In the design of such links, because they can be made synchronous with respect to the timing epochs of the symbols of the various signals, it is possible to employ orthogonal spreading sequences.

Note, however, that if a multicarrier waveform is used such that $M$ subcarriers span the spread bandwidth, then, relative to a wideband single carrier DS system, the number of orthogonal functions is reduced by a factor of $M$ (assuming the system is designed just to maintain orthogonality within each subband). Hence, there is the potential for significantly more multiple access interference on the forward link because of the use of multicarrier signaling, compared to the multiple access interference of a single carrier system, which only occurs because of multipath and out-of-cell interference. To help alleviate this situation, one can consider a hybrid scheme of the type described in [46]. Each transmitter's data stream is now divided into, say, $R$ parallel streams, where $R=M / H$, and both $R$ and $H$ are integers. By doing this, one can now increase the processing gain of each carrier by a factor of $R$, since each symbol has its duration increased by the factor of $R$. The penalty for doing this is a loss in frequency diversity. That is, whereas the degree of frequency diversity in the original multicarrier design is determined by the total number of carriers, i.e., $M$, in the hybrid system it is determined by $H$ (or $M / R$ ). Thus, one now has a tradeoff be- tween the number of orthogonal waveforms and the amount of frequency diversity. This tradeoff is quantified in [46].

\section{Multicode CDMA}

Yet another possible change in the design of a CDMA network is to incorporate what is referred to as multicode CDMA [2], [23], [38], [67]. Perhaps the key motivation for multicode CDMA is that of increasing the information rate over a given spread bandwidth, an objective which is fundamental to future generation CDMA systems. An additional reason is to allow for the flexibility of multiple data rates. If one attempts to increase the data rate simply by decreasing the information symbol duration, while simultaneously keeping spread bandwidth constant, the processing gain necessarily decreases. Indeed, such systems are typically referred to as variable processing gain (VPG) systems. As an alternative to a VPG waveform, one can assign multiple spreading sequences to any given user. If these spreading sequences are orthogonal, the self-interference caused by them is reduced. Clearly, there are tradeoffs in the use of these two techniques to increase the data rate.

With respect to VPG systems, consider the situation at high data rates, wherein the processing gain can be very small. The result of a small processing gain is, among other things, a reduction in the effective order of diversity that the receiver can achieve. This is because a reduced processing gain results in increased correlation among the taps of a rake receiver. Alternately, the multicode system suffers from increased interference, since each user now transmits multiple waveforms simultaneously, and each of those waveforms is affected by the multipath on the channel. Further, because the multicode waveform does not have a constant envelope, it is adversely affected by any nonlinearities in the system, such as a saturating amplifier. For a quantitative assessment of this latter effect, as well as the results of using various signal constellations, see [33].

\section{AdDitional EnHancements to WCDMA System DESIGN}

\section{A. Packet Transmission in Cellular Wireless Systems}

A fundamental goal for future wireless CDMA systems is to allow for high speed data transmission over packet switched wireless networks, as opposed to the conventional circuit switched systems that have been designed for voice traffic. However, if one wants efficient transmission of, say, an individual datagram, the architecture of the current NCDMA systems might not be efficient, because it uses a pilot channel that is on continuously, and thus is creating interference even when packets of a given user are not being transmitted (which might be most of the time). Thus, consideration has been given to replacing a pilot that is code division multiplexed with the data, with a header that is time division multiplexed with the data (see, e.g., [1], [6], and [71]).

However, because the standard means to achieve coarse acquisition is to use a serial correlation receiver, the combination of it and the time division multiplexed header might not be effective. That is, while such a receiver is simple to implement, it leads to long acquisition times, since all unknown phase positions of the incoming waveform must be searched in series. 
To remedy the above situation, WCDMA systems are being considered which replace the serial correlator with a passive matched filter [29], [71]. As is well known, a matched filter can achieve coarse acquisition almost as fast as a parallel correlator bank, wherein each component of the correlator bank is matched to one of the unknown phase positions of the spreading sequence.

Note that the use of matched filter technology yields a variety of additional benefits. For example, independent of whether packet transmission is to be employed, the substitution of a single correlator with a matched filter results in decreased acquisition time. In turn, this decreased acquisition time can be useful in preserving battery life in a handheld device; when a signal is not being received, many of the ASIC's in the receiver can be periodically turned off (for a predetermined duration of time) and then turned on to rapidly search for a signal.

In the context of wideband versus narrowband CDMA, a matched filter is more beneficial to WCDMA, since it allows easy identification of the multipath components. That is, since the matched filter is a passive device, any multipath component that arrives while the filter is matched to a particular spreading pattern results in a voltage peak at the output of the filter indicating the timing epoch of that multipath component. Thus, there is no need to be searching for available multipath returns upon which to track. Since a larger spread bandwidth typically results in a larger number of resolvable paths, matched filter technology is especially useful to wideband systems.

\section{B. Multiple Access Interference Suppression}

A second possible enhancement being considered for WCDMA is to employ signal processing techniques on the uplink to suppress multiple access interference (MAI), an idea originally proposed by Verdu in [80]. There are many such techniques, and the type of structure that seems most likely to be used initially belongs to the class of multiuser receivers which perform interference cancellation (see, e.g., [19] and [56]). That is, they make an estimate of the dominant interfering terms as seen by a given user, and then subtract those estimates from the test statistic of the user-of-interest. Indeed, experimental results have been generated for this type of interference suppression technique, and [68] and [35] describe some of those results. Because interference cancellation requires that the receiving terminal despread and demodulate all signals for which it will attempt cancellation, the use of the scheme is most appropriate for the uplink, wherein the base station already has to perform these functions.

Given the extensive literature already in existence regarding the description of these schemes, as well as their performance under various conditions, neither of these topics will be repeated in this paper (the interested reader can find an in-depth treatment, as well as an extensive list of references, in [81]). Rather, in keeping with the theme of this paper, we will discuss the impact of spread bandwidth on the performance of multiple access interference suppression. This is perhaps most easily illustrated with a class of receiver structures known as single user receivers. They are often designed for interference suppression based upon a minimum mean-squared error (MMSE) approach, and implemented with a standard tapped delay line. Further, they are amenable to peer-to-peer architectures, where power control is not feasible.

Since knowledge of only the parameters of the user-of-interest is required, such a receiver has the obvious advantage of simplicity relative to, say, interference cancellation (since one no longer needs to know anything about the other users' signals). It also has the advantage of ease of adaptation, since standard algorithms such as least mean squares (LMS) or recursive least squares (RLS) can be employed. On the hand, it suffers from the disadvantage of requiring the use of short spreading sequences, since the interference must have cyclostationary statistics in order for the adaptation algorithms to function. That is, the statistics of the MAI should be periodic in the update interval, which is typically once every symbol.

By definition, a short spreading sequence is one whose period equals the duration of the symbol being spread, whereas a long spreading sequence is one whose period spans multiple symbols. In the current cellular CDMA designs, long spreading sequences are employed, and the rationale is twofold. Since a short spreading sequence implies that successive symbols are spread with the same chip pattern, if a given set of users is in a relatively static situation (e.g., everyone caught in a traffic jam), the interference pattern seen by any specific receiver is the same from bit to bit. Thus, some users might be caught in particularly disadvantageous situations relative to those of other users. However, with a long spreading sequence, the interference patterns of all users vary bit to bit, and thus an averaging effect is seen. Further, there are only a limited number of short spreading sequences with good cross-correlation properties, and so the issue of code reuse (analogous to frequency reuse) has to be addressed with the use of short spreading sequences.

Thus, even before considering the effect of spread bandwidth, the question that arises is how well short spreading sequences function when used in conjunction with single-user MMSE receivers (i.e., do we want to consider the use of such sequences for any spread bandwidth?). In [55], that question is addressed. A reference cell, in which all mobile units are assigned deterministic short spreading sequences, is surrounded by two layers of other cells in which each mobile, of each cell, has its spreading sequence chosen randomly. This allows for, statistically, the possibility of reusing the same spreading sequence in a neighboring cell that had been assigned to a mobile in the cell-of-interest. In Fig. 7, taken from [55], the results of this study can be seen. There are three curves in Fig. 7, each one of which is a histogram of signal-to-noise-plus-interference ratios. Note that, in the absence of MAI suppression, the average values of the histograms for either short or long sequences are roughly the same, although the variance of the histogram corresponding to the long sequences is smaller than that corresponding to the short sequences (thus indicating that the use of the long sequences is preferable). However, once the short sequences are used in conjunction with MMSE MAI suppression, the histogram shifts noticeably to the right, yielding the best performance of all three cases.

If one then is, in fact, using a set of short spreading sequences, it is relevant to determine how well a single-user MMSE receiver performs, in particular with respect to WCDMA. Toward 


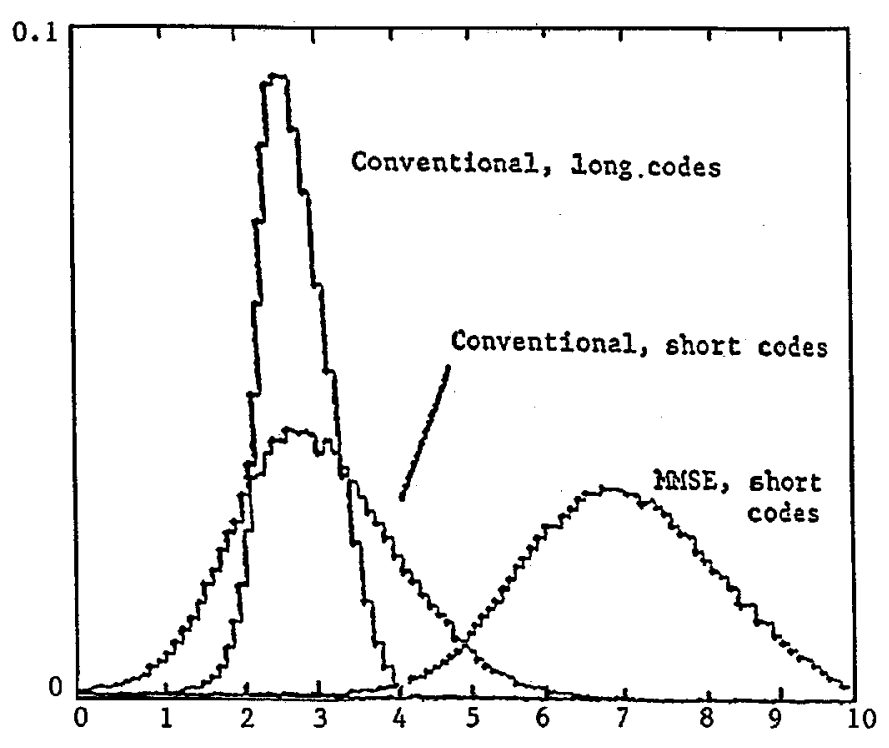

Fig. 7. Distribution of the average SNR in decibels.

that end, consider results from [48] and [27], where [48] corresponds to an uncoded system, and [27] corresponds to one where both convolutional and trellis coding are considered. In both scenarios, it is found that, although the use of a single-user, MMSE suppression filter can be very beneficial for a signal experiencing flat fading (i.e., for an NCDMA network), the combined use of the MMSE receiver with WCDMA is not always worthwhile. Rather, it is a function of whether or not the fading on the multiple resolvable paths of the interfering waveforms can be tracked by the MMSE receiver. This, in turn, is a function of how rapidly the channel is changing (i.e., it is a function of how large is the Doppler spread on the channel). For small Doppler spreads, the channel changes slowly, the multipath can be tracked, and the MMSE yields a sizable improvement in performance over that of a simple correlation receiver. For large Doppler spreads, the multipath cannot be tracked and the MMSE receiver can lose its effectiveness. However, when a severe near-far problem exists, even with a large Doppler spread, the improvement due to the presence of the suppression filter can be substantial. Such scenarios tend to exist in either peer-to-peer networks, or in cellular systems where accurate power control cannot be maintained.

\section{Transmit Diversity}

Consider now the use of the multiple transmit antennas on the forward link. Such a system design clearly allows for the possibility of a diversity gain on a fading channel. However, the amount of the gain is a function of a variety of parameters, including the number of antennas, the correlation of the signals from the separate antennas at the receiver, the Doppler spread on the channel, and the spread bandwidth. As with any type of diversity, the gain achievable increases as the number of diversity elements increases, and decreases as the correlation among those elements increases. Further, one experiences diminishing returns fairly rapidly as the number of diversity elements increases.

Thus, with respect to CDMA [34], [37], it is to be expected that antenna diversity will yield the most benefit when used on a slow fading channel in conjunction with narrowband CDMA. With respect to fade rate, assuming that coding and interleaving are part of the system design, a rapidly varying channel (i.e., one with a large Doppler spread), the combination of coding and interleaving provides time diversity, thus reducing the need for (and the corresponding benefit of) spatial diversity. Alternately, if the fade rate is low, the amount of time diversity is decreased, and so the benefit of the spatial diversity is increased.

In an analogous sense, a WCDMA system offers more path diversity than does a narrowband one. Thus, the need for multiple transmit antennas to provide spatial diversity in WCDMA is less than that in NCDMA. The above qualitative statements are illustrated quantitatively in references such as [34] and [37], wherein it is shown, for example, that the improvement in performance in going from a single transmit antenna to two transmit antennas is greater for a receiver that can resolve just a single path than for a receiver capable of resolving two paths.

\section{CONCLUSION}

In this paper, a discussion of some of the essential features of WCDMA has been presented. Among the topics which have been emphasized are the following: One of the key reasons for transitioning from NCDMA to WCDMA is the enhanced performance that one achieves over a wireless channel by ensuring that one is virtually always in a situation whereby the fading channel appears frequency-selective to the transmitted waveform. How one achieves the wider spread is not unique, and some of the specific attributes of one relatively new technique, namely MC CDMA, were discussed. Other topics discussed were the use of multicode CDMA, the use of transmit diversity, the considerations involved for CDMA systems to transition from a circuit switched environment to a packet switched environment, and the use of MAI suppression as a natural means of enhancing the inherent multiple access capability of CDMA.

\section{ACKNOWLEDGMENT}

The author would like to thank Prof. M. B. Pursley for his extremely helpful review, and the three anonymous reviewers for their many useful comments.

\section{REFERENCES}

[1] S. Abeta, M. Sawahashi, and F. Adachi, "Performance comparison between time-multiplexed pilot channel and parallel pilot channel for coherent rake combining in DS-CDMA mobile radio," IEICE Trans. Commun., vol. E81-B, no. 7, pp. 1417-1425, July 1998.

[2] F. Adachi, K. Ohno, A. Higashi, R. Dohi, and Y. Okumura, "Coherent multicode DS-CDMA mobile radio access," IEICE Trans. Commun., vol. E79B, pp. 1316-1325, Sept. 1996.

[3] F. Adachi, M. Sawahashi, and H. Suda, "Wideband DS-CDMA for nextgeneration mobile communications systems," IEEE Commun. Mag., pp. 56-69, Sept. 1998.

[4] J. M. Aein, "Multiple access to a hard-limiting communication-satellite repeater," IEEE Trans. Space Electron. Telemetry, vol. SET-10, pp. 159-167, Dec. 1964.

[5] D. R. Anderson and P. A. Wintz, "Analysis of a spread-spectrum multiple-access system with a hard limiter," IEEE Trans. Commun. Technol., vol. COM-17, pp. 285-290, Apr. 1969.

[6] H. Andoh, M. Sawahashi, and F. Adachi, "Channel estimation filter using time-multiplexed pilot channel for coherent RAKE combining in DS-CDMA mobile radio," IEICE Trans. Comm., vol. E81-B, no. 7, pp. 1517-1526, July 1998. 
[7] P. A. Bello, "Performance of some RAKE modems over the nondisturbed wide band HF Channel," in Proc. 1998 IEEE Military Commun. Conf., pp. 4.3.1-4.3.7.

[8] H. Blasbalg, "A comparison of pseudo-noise and conventional modulation for multiple-access satellite communications," IBM J., vol. 9, pp. 241-255, July 1965

[9] D. E. Borth and M. B. Pursley, "Analysis of direct-sequence spread spectrum multiple-access communications over Rician fading channels," IEEE Trans. Commun., vol. COM-27, pp. 1566-1577, Oct. 1979.

[10] A. Brajal and A. Chouly, "Compensation of nonlinear distortions for orthogonal multicarrier schemes using predistortion," in Proc. Globecom'94, vol. 3, San Francisco, CA, Nov. 1994, pp. 1909-1914.

[11] A. Chockalingam and L. B. Milstein, "Open-loop power control performance in DS-CDMA networks with frequency selective fading and nonstationary base stations," ACM Wireless Networks J., no. 4, pp. 249-261, 1998.

[12] A. Chockalingam, L. B. Milstein, P. Dietrich, and R. R. Rao, "Performance of closed-loop control in DS-CDMA cellular systems," IEEE Trans. Veh. Technol., vol. 47, pp. 774-789, Aug. 1998.

[13] A. Chouly, A. Brajal, and S. Jourdan, "Orthogonal multicarrier techniques applied to direct sequence spread spectrum CDMA systems," in Proc. Globecom'98, vol. 3, Houston, TX, Nov. 1993, pp. 1723-1728.

[14] V. M. DaSilva and E. S. Sousa, "Multicarrier orthogonal CDMA signals for quasi-synchronous communication systems," IEEE J. Select. Areas Commun., vol. 12, pp. 842-852, June 1994.

[15] M. Davis and L. B. Milstein, "Filtered spreading sequences for interference avoidance," in Proc. Int. Conf. Universal Personal Commun., 1992.

[16] S. Dhar and B. D. Perry, "Equalized megahertz-bandwidth HF channels for spread-spectrum communications," in Proc. 1982 IEEE Military Commun. Conf., pp. 29.5-1-29.5-5.

[17] T. Dohi, Y. Okumura, and F. Adachi, "Effects of spreading chip rates on transmit power distribution in power-controlled DS-CDMA reverse link," Electron. Lett., vol. 33, no. 6, pp. 447-448, Mar. 1997.

[18] — "Further results on field experiments of coherent wideband DS-CDMA mobile radio," IEICE Trans. Commun., vol. E81-B, no. 6, pp. 1239-1247, June 1998.

[19] A. Dual-Hallen, J. Holtzman, and Z. Zvonar, "Multiuser detection for CDMA systems," IEEE/ACM Personal Commun., vol. 2, pp. 46-58, 1995.

[20] T. Eng and L. B. Milstein, "Comparison of hybrid FDMA/CDMA systems in frequency selective Rayleigh fading," IEEE J. Select. Areas Commun., vol. 12, pp. 938-951, June 1994

[21] — "Partially coherent DS-SS performance in frequency selective multipath fading," IEEE Trans. Commun., vol. 45, pp. 110-118, Jan. 1997.

[22] T. Eng, A. Chockalingam, and L. B. Milstein, "Capacities of FDMA/CDMA systems in the presence of phase noise and multipath Rayleigh fading," IEEE Trans. Commun., vol. 46, pp. 997-999, Aug. 1998.

[23] P. K. Enge and D. V. Sarwate, "Spread-spectrum multiple-access performance of orthogonal codes: Linear receivers," IEEE Trans. Commun., vol. COM-35, pp. 1309-1319, Dec. 1987.

[24] R. Eschenbach, "Applications of spread-spectrum radio to indoor data communications," in Proc. 1982 IEEE Military Commun. Conf., pp. $34.5-1-34.5-3$

[25] K. Frazel and L. Papke, "On the performance of convolutionally-coded CDMA/OFDM for mobile communication system," in Proc. PIMRC/93, Yokohama, Japan, Dec. 1993, p. D3.2.1-5.

[26] K. G. Filis and S. C. Gupta, "Coexistence of cellular CDMA and FSM Interference suppression using filtered PN sequences," in Proc. IEEE 1993 Global Telecommun. Conf., pp. 898-902.

[27] J. R. Foerster, "The Performance of Matched-Filter and MMSE Receivers for DS-CDMA Systems in Multipath Fading Channels," Ph.D. dissertation, UCSD, 1998

[28] P. Freret, R. Eschenbach, D. Crawford, and P. Braisted, "Applications of spread-spectrum radio to wireless terminal communications," in Proc. 1980 IEEE National Telecommunications Conf., pp. 69.7.1-69.7.4.

[29] S. Fukumoto, M. Sawahashi, and F. Adachi, "Matched filter-based RAKE combiner for wideband DS-CDMA mobile radio," IEICE Trans., vol. E81-B, no. 7, pp. 1384-1391, July 1998

[30] J. H. Gass Jr., D. L. Noneaker, and M. B. Pursley, "On the spectral efficiency of a power-controlled CDMA mobile personal communication system," IEEE J. Select. Areas Commun., vol. 14, pp. 559-569, Apr. 1996.

[31] L. D. Gottesman and L. B. Milstein, "The coarse acquisition performance of a CDMA overlay system," IEEE J. Select. Areas Commun., vol. 14 , pp. $1627-1635$, Oct. 1996
[32] L. J. Greenstein, V. Erceg, Y. S. Yeh, and M. V. Clark, "A new path-gain/delay-spread propagation model for digital cellular channels," IEEE Trans. Veh. Technol., vol. 46, pp. 477-485, May 1997.

[33] N. Guo and L. B. Milstein, "Uplink performance evaluation of multicode DS/CDMA systems in the presence of nonlinear distortions," IEEE $J$. Select. Areas Commun., vol. 18, pp. 1418-1428, Aug. 2000.

[34] T. Heikkinen and A. Hottinen, "On downlink power control and capacity with multi-antenna transmission," in Proc. 1998 48th IEEE Veh. Technol. Conf., vol. 1, May 1998, pp. 475-479.

[35] H. Higuchi, H. Andoh, K. Okawa, M. Sawahashi, and F. Adachi, "Field experiments on pilot symbol-assisted coherent multistage interference canceller for DS-CDMA mobile radio," in Proc. 4th CDMA Int. Conf. Exhibition (CIC'99), Seoul, Korea, Sept. 1999, pp. 190-194.

[36] J. M. Holtzman and L. M. A. Jalloul, "Rayleigh fading effect reduction with wideband DS/CDMA signals," IEEE Trans. Commun., vol. 42, pp. 1012-1016, Apr. 1994.

[37] A. Hottinen and R. Wichman, "Transmit diversity antenna selection in CDMA downlink," in Proc. 1998 IEEE 5th Int. Symp. Spread Spectrum Techniques Appl., vol. 3, Sept. 1998, pp. 767-770.

[38] C.-L. I and R. D. Gitlin, "Multi-code CDMA wireless personal communications networks," in Proc. IEEE Int. Conf. Commun. (ICC'95), vol. 2, Seattle, WA, pp. 1060-1064.

[39] A. N. Ince, "Code division multiplexing for satellite systems," in Communication Systems and Random Process Theory. ser. NATO Advanced Study Institute Series, J. K. Skwirzynski, Ed. Alphen aan den Rijn, Netherlands: Sijthoff and Noordhoff International, 1978, pp. 821-857.

[40] A. Jalali and P. Mermelstein, "Effect of diversity, power control, and bandwidth on the capacity of microcellular CDMA systems," IEEE J. Select. Areas Commun., vol. SAC-12, pp. 952-961, June 1994.

[41] L. M. A. Jalloul and J. M. Holtzman, "Multipath fading effects on wide-band DS/CDMA signals: Analysis, simulation, and measurements," IEEE Trans. Veh. Technol., vol. 43, pp. 801-807, Aug. 1994.

[42] S. Kondo and L. B. Milstein, "On the use of multicarrier direct sequence spread spectrum systems," in Proc. 1993 IEEE Military Commun. Conf., Oct. 1993 , pp. $52-56$

[43] _ - "Multicarrier DS CDMA systems in the presence of partial-band interference," in Proc. IEEE Military Commun. Conf., Oct. 1994, pp. 588-599.

[44] — , "Performance of multicarrier DS CDMA systems," IEEE Trans. Commun., vol. 44, pp. 238-246, Feb. 1996.

[45] D. Lee and L. B. Milstein, "Analysis of multicarrier DS-CDMA code acquisition system," IEEE Trans. Commun., vol. 47, pp. 1233-1244, Aug. 1999.

[46] - "Comparison of multicarrier DS CDMA broadcast systems in a multipath fading channel," IEEE Trans. Commun., vol. 47, pp. 1897-1904, Dec. 1999.

[47] J. Low and S. M. Waldstein, "A direct sequence spread-spectrum modem for wideband HF channels," in Proc. 1982 IEEE Military Commun. Conf., pp. 29.6-1-29.60-6.

[48] S. L. Miller, M. L. Honig, and L. B. Milstein, "Performance analysis of MMSE receivers for DS-CDMA in frequency selective fading channels," IEEE Trans. Commun. submitted for publication.

[49] L. B. Milstein and D. L. Schilling, "The CDMA overlay concept," in Proc. IEEE 4th Int. Symp. Spread Spectrum Techniques Appl., Sept. 1996, pp. 476-480.

[50] L. B. Milstein, D. L. Schilling, R. L. Pickholtz, V. Erzeg, M. Kullback, E. Kanterakis, D. Fishman, W. H. Biederman, and D. Salerno, "On the feasibility of a CDMA overlay for personal communications networks," IEEE J. Select. Areas Commun., vol. 10, pp. 655-668, May 1992.

[51] B. B. Mohebbi, A. H. Aghvami, and W. G. Chambers, "Broadband channel modeling and performance analysis," in Proc. 3rd IEEE ISSSTA'94, vol. 2, Oulu, Finland, July 4-6, 1994, pp. 637-641.

[52] D. L. Noneaker and M. B. Pursley, "On the chip rate of CDMA systems with doubly selective fading and rake reception," IEEE J. Select. Areas Commun., vol. 12, pp. 853-861, June 1994.

[53] — "Selection of spreading sequences for direct-sequence spread-spectrum communications over a doubly selective fading channel," IEEE Trans. Commun., vol. 42, pp. 3171-3177, Dec. 1994

[54] _ "The effects of sequence selection on DS spread spectrum with selective fading and rake reception," IEEE Trans. Commun., vol. 44, pp. 229-237, Feb. 1996.

[55] S. Parkvall, "Long vs. short spreading codes in cellular DS-CDMA," in Proc. ISSSTA 1998.

[56] P. Patel and J. Holtzman, "Analysis of a simple successive interference cancellation scheme in a DS/CDMA system," IEEE J. Select. Areas Commun., vol. 12, pp. 796-807, June 1994. 
[57] B. D. Perry, "A new wideband HF technique for MHz-bandwidth spread-spectrum radio communications," IEEE Commun. Mag., pp. 28-36, Sept. 1983.

[58] R. Price and P. E. Green Jr., "A communication technique for multipath channels," Proc. IRE, vol. 46, pp. 555-570, Mar. 1958.

[59] M. B. Pursley, "Performance evaluation for phase-coded spread-spectrum multiple-access communication-Part I: System analysis," IEEE Trans. Commun., vol. COM-25, pp. 795-799, Aug. 1977.

[60] B. J. Rainbolt and S. L. Miller, "Multicarrier CDMA for cellular overlay systems," IEEE J. Select. Areas Commun., vol. 17, pp. 1807-1814, Oct. 1999.

[61] R. R. Rick and L. B. Milstein, "Parallel acquisition in mobile DS-CDMA systems,” IEEE Trans. Commun., vol. 45, pp. 1466-1476, Nov. 1997.

[62] _ - "Optimal decision strategies for acquisition of spread spectrum signals in frequency selective fading channels," IEEE Trans. Commun., vol. 46, pp. 686-694, May 1998.

[63] D. N. Rowitch and L. B. Milstein, "Coded multicarrier code division multiple access," in Proc. IEEE Int. Symp. Inform. Theory, Sept. 1995, p. 23.

[64] — "Convolutionally coded multicarrier DS CDMA systems in a multipath fading channel-Part I: Performance analysis," IEEE Trans. Commun., vol. 47, pp. 1570-1582, Oct. 1999.

[65] - "Convolutionally coded multicarrier DS CDMA systems in a multipath fading channel-Part II: Narrowband interference suppression," IEEE Trans. Commun., vol. 47, pp. 1729-1736, Nov. 1999.

[66] L. A. Rusch and H. V. Poor, "Narrowband interference suppression in CDMA spread spectrum communications," IEEE Trans. Commun., Apr. 1994.

[67] S. Sasaki, H. Kikuchi, H. Watanabe, and J. Zhu, "Performance evaluation of parallel combinatory SSMA systems in Rayleigh fading channel," in Proc. IEEE 3rd Int. Symp. Spread Spectrum Techniques Appl. (ISSSTA'94), vol. 1, Oulu, Finland, pp. 198-202.

[68] M. Sawahashi, H. Andoh, K. Higuchi, and F. Adachi, "Experiments on coherent multistage interference canceller for DS-CDMA mobile radio," in Proc. PIMRC'98, Sept. 1998, pp. 491-496.

[69] D. L. Schilling, "Spread Spectrum CDMA Communications Systems Microwave Overlay,", Nov. 3, 1992. U.S. Patent 5161168.

[70] D. L. Schilling et al., "Broadband CDMA overlay," Int. J. Wireless Inform. Networks, vol. 2, no. 4, pp. 197-221, Oct. 1995.

[71] D. L. Schilling, J. Garodnick, L. B. Milstein, and K. K. Lee, "Wideband CDMA for packet switched communications," in Proc. 2nd CDMA Int. Conf. Exhibition, vol. II, Seoul, Korea, Oct. 1997, pp. 243-245.

[72] A. Shah and A. M. Haimovich, "Performance of space-time receiver architectures for CDMA overlay of narrowband waveforms for personal communication systems," in Proc. 1997 IEEE Int. Conf. Commun., June 1997, pp. 314-318

[73] L. Shapira and C. E. Wheatley, "Channel based optimum bandwidth for spread spectrum land cellular radio," in Proc. 1992 IEEE Int. Symp. Personal, Indoor Mobile Radio Commun., Boston, MA, Oct. 1992, pp. 199-204.

[74] R. Skaug and J. L. Robinson, "Operation of portable HF CDMA networks with controlled transmitter powers," IEE Proc., pp. 467-474, Dec. 1982.

[75] E. A. Sourour and M. Nakagawa, "Performance of orthogonal multicarrier CDMA in a multipath fading channel," IEEE Trans. Commun., vol. 44, pp. 356-367, Mar. 1996.

[76] Special issue IMT-2000: Standards efforts of the ITU, IEEE Personal Commun., vol. 4, Aug. 1997.

[77] — , "Wideband CDMA," IEEE Commun. Mag., vol. 36, Sept. 1998. special Issue.

[78] G. L. Turin, "Introduction to spread-spectrum antimultipath techniques and their application to urban digital radio," in Proc. IEEE, vol. 68, Mar. 1980.

[79] - "The effects of multipath and fading on the performance of direct-sequence CDMA systems," IEEE J. Select. Areas Commun., pp. 597-603, July 1984.
[80] S. Verdu, "Minimum probability of error for asynchronous Gaussian multiple-access channels," IEEE Trans. Inform. Theory, vol. IT-32, pp. 85-96, January 1986.

[81] _ Multiuser Detection. New York: Cambridge Press, 1998.

[82] A. J. Viterbi, "CDMA-Principles of Spread Spectrum Communication, ser. Wireless Communications Series. Reading, MA: Addison-Wesley, 1995.

[83] J. Wang and L. B. Milstein, "CDMA overlay situations for microcellular mobile communications," IEEE Trans. Commun., pp. 603-614, Feb. 1995.

[84] P. Wei, T. Soni, J. R. Zeidler, W. H. Ku, and P. K. Das, "Adaptive interference suppression for CDMA based PCN systems," in Proc. IEEE Int. Conf. Commun., Geneva, Switzerland, May 1993.

[85] W. Xu and L. B. Milstein, "On the performance of multicarrier RAKE systems," in Proc. 1997 IEEE Global Telecommun. Conf., vol. 1, Nov. 1997, pp. 295-299.

[86] - "On the use of interference suppression to reduce intermodulation distortion in multicarrier CDMA systems," IEEE Trans. Commun., submitted for publication.

[87] K. Yao, "Error probability of asynchronous spread spectrum multiple access communication systems," IEEE Trans. Commun., vol. COM-25, pp. 803-809, Aug. 1977.

[88] N. Yee, J. P. Linnartz, and G. Fettweis, "Multicarrier CDMA in indoor wireless radio," in Proc. PIMRC'93, Yokohama, Japan, Dec. 1993, p. D1.3.1-5.

[89] R. E. Ziemer and N. Nadgauda, "Effect of correlation between subcarriers of an MCM/DSSS communication system," presented at the Proc. VTC, 1996

[90] R. E. Ziemer, B. R. Vojcic, L. B. Milstein, and J. G. Proakis, "Effects of carrier tracking in RAKE reception of wide-band DSSS in Rician fading," IEEE Trans. Microwave Theory Tech., vol. 47, pp. 681-686, June 1999.

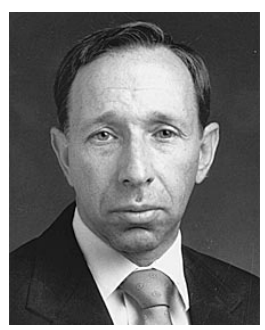

Laurence B. Milstein (S'66-M'68-SM'75-F'85) received the B.E.E. degree from the City College of New York, New York, NY, in 1964, and the M.S. and Ph.D. degrees in electrical engineering from the Polytechnic Institute of Brooklyn, Brooklyn, NY, in 1966 and 1968, respectively.

From 1968 to 1974 , he was employed by the Space and Communications Group of Hughes Aircraft Company, and from 1974 to 1976, he was a member of the Department of Electrical and Systems Engineering, Rensselaer Polytechnic Institute, Troy, NY. Since 1976, he has been with the Department of Electrical and Computer Engineering, University of California at San Diego, La Jolla, CA, where he is a Professor and former Department Chairman, working in the area of digital communication theory with special emphasis on spread-spectrum communication systems. He has also been a consultant to both government and industry in the areas of radar and communications.

Dr. Milstein was an Associate Editor for Communication Theory for the IEEE TRANSACTIONS ON COMMUNICATIONS, an Associate Editor for Book Reviews for the IEEE TRANSACTIONS ON INFORMATION THEORY, and an Associate Technical Editor for the IEEE COMMUNICATIONS MAGAZINE, and the Editor-in-Chief of the IEEE Journal on SELECTED AREAS IN COMMUNICATIONS. He was the Vice President for Technical Affairs in 1990 and 1991 of the IEEE Communications Society, and has been a member of the Board of Governors of both the IEEE Communications Society and the IEEE Information Theory Society. He has been a member of the IEEE Fellows Selection Committee since 1996, and he currently is the chair of that committee. He is also the chair of ComSoc's Strategic Planning Committee. He is a Fellow of the IEEE, a recipient of the 1998 Military Communications Conference Long Term Technical Achievement Award, a 1999 UCSD Distinguished Teaching Award, and a IEEE Third Millenium Medal. 